\title{
Research Paper \\ The Relationship Between Tendency to Substance Abuse and Resilience and Academic Procrastination in Secondary School Students
}

\author{
Sedigheh Ahmadi ${ }^{1}\left(\mathbb{C},{ }^{*}\right.$ Saeed Toulabi ${ }^{2}$ (D), Hossein Ilanloo ${ }^{1}$ (1)
}

1. Department of Counselling, Faculty of Psychology, Kharazmi University, Tehran, Iran.

2. Department of Educational Psychology, Faculty of psychology, Kharazmi University, Tehran, Iran.

\begin{tabular}{|c|c|}
\hline $\begin{array}{l}\text { Use your device toscan } \\
\text { and read the article online }\end{array}$ & Crtation: Ahmadi S. Toulabi S. Ilanloo H. [The Relationship Between Tendency to Substance Abuse and Resilience and Aca- \\
\hline 口ipera & demic Procrastination in Secondary School Students (Persian)]. Journal of Arak University of Medical Sciences (JAMS). 2020; \\
\hline hetsis & 23(1):108-117. https://doi.org/10.32598/JAMS.23.1.5972.1 \\
\hline 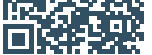 & doi https://doi.org/10.32598/JAMS.23.1.5972.1 \\
\hline
\end{tabular}

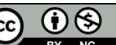

Article Info:

Received: 27 Aug 2019

Accepted: 09 Dec 2019

Available Online: 01 Apr 2020

Key words:

Academic procrastination, Substance abuse tendency, Resilience

\section{ABSTRACT}

Background and Aim Adolescent students are at high risk for substance abuse. The tendency to substance abuse in students is of significant importance. Therefore, this study aimed to investigate the relationship between the tendency to substance abuse based on resilience and procrastination in high school students.

Methods \& Materials This was a cross-sectional and correlational study. The statistical population consisted of high-school male students in district 12 of Tehran City, Iran, in the academic year of 2017-2018. The statistical sample consisted of 149 individuals who were selected using a multistage cluster sampling technique. The data collection instruments were Conner and Davidson's Persistence Questionnaire (2003), Solomon and Roth Blum (1984), and Golparvar Substance Abuse Tendency Questionnaire (2003). Pearson's correlation coefficient and regression analysis were used for data analysis.

Ethical Considerations This study was approved by the Research Ethics Committee of Kharazmi University (Code: IR.KHU.REC1397.29).

Results The current study results suggested a significant relationship between resilience and the tendency to substance abuse $(P<0.05)$. There was a significant and positive relationship between academic procrastination and the tendency to substance abuse $(P<0.05)$ in the study samples. Academic resilience and procrastination could explain $54 \%$ of the variance in substance abuse tendency in the investigated adolescents. Resiliency $(-0.556)$ and academic procrastination $(-0.436)$ played a significant role in predicting the tendency of substance abuse in adolescents.

Conclusion Academic resilience and procrastination predicted a tendency for substance abuse in secondary school students. Therefore, these risk factors need to be considered in preventive measures and interventions for this age group.

\section{* Corresponding Author:}

Saeed Toulabi, PhD.

Address: Department of Educational Psychology, Faculty of psychology, Kharazmi University, Tehran, Iran

Tel: +98 (912) 5840062

E-mail: saeedtoulabi@yahoo.com 


\section{Extended Abstract}

\section{Introduction}

1

Significant human issue that vastly affected the biopsychosocial and economic dimensions of life is substance abuse and dependence. Moreover, it has inflicted irreparable damages on the human body at micro and macro levels. Substance abuse is among the problems of human society, i.e., increasing daily [1]. An affecting factor on adolescents' tendency to abuse drugs is their resilience. Resilience includes a set of individual and psychological resources with which a person could survive in difficult situations and not be harmed; even in experiencing difficult or critical situations, he/she could improve his/her personality. In other words, resilience could be assumed as a psychological concept that seeks to explain individuals' coping styles in unexpected situations. However, resilience not only increases a person's ability to tolerate and adapt to a problem, but more importantly, maintains mental health, and even promotes it. Resilience enables individuals to face the difficulties and hardships of life and work, without being harmed, and even to use these opportunities for personality growth [2].

Another concept explored in this study was academic procrastination. Academic procrastination has been defined as delaying studying for exams, delaying to perform homework, and failing to register for classes on time. Besides, this concept has attracted numerous researchers' attraction in recent years [8]. Academic procrastination is a frequent problem among students [9]. Although procrastination may occur in all activities of daily living, it is more prevalent in the field of homework conduction. Procrastination of homework is among the main reasons why learners fail to learn and have academic achievement. Academic procrastination is associated with academic assignments and could be described as delaying academic assignments for various reasons.

Researchers have extensively investigated factors associated with students 'negligence, or more precisely, students' academic procrastination. Accordingly, various psychological, family and social determinants, like internal control have been determined. External, general self-efficacy and self-regulation [11], and educational styles [12] were examined in this area.

\section{Materials and Methods}

This was a cross-sectional and analytical correlational study. The number of statistical samples using Cochran's formula was calculated as 150 individuals. The study participants were selected using a multistage cluster sampling method. The study samples completed the study questionnaires. Inclusion criteria were as follows: being in the second year of high-school, unmarried, and aged 14-17 years. Exclusion criteria were being an unwillingness to participate in the research and providing uncompleted questionnaires. Due to the distortion of one of the questionnaires, a statistical sample of 149 individuals was finally considered. The instruments used in this study were Conner and Davidson's Persistence Questionnaire (2003) [14], Solomon and Roth Blum [15], and Golparvar Substance Abuse Tendency Questionnaire [17]. Pearson's correlation coefficient and regression analysis were used for data analysis.

\section{Results}

The correlation coefficient data of the research variables revealed that the degree of correlation obtained between resilience and substance abuse tendency was equal to -0.609 $(\mathrm{P}<0.01)$. Furthermore, the correlation between academic procrastination and substance abuse was calculated as $0.394(\mathrm{P}<0.01)$. In addition, the correlation between resilience and academic achievement was measured as -0.547 $(\mathrm{P}<0.01)$ (Table1).

As per Table 2, with the simultaneous entry of the predictor variables into the equation, the coefficient of explanation was calculated as 0.54 . These variables were used as predictors to examine the effect of the studied variables in predicting substance abuse.

Furthermore, the prediction ratios for substance abuse based on student resilience and academic procrastination suggested that these variables could explain $54 \%$ of the variance in substance abuse in the studied students. More-

Table 1. Mean \pm SD values of substance abuse tendency, academic procrastination, and resilience

\begin{tabular}{cccc}
\hline Variable & Mean \pm SD & Min.t & Max. \\
\hline Substance abuse tendency & $13.18 \pm 8.03$ & 2 & 29 \\
Academic procrastination & $52.14 \pm 13.42$ & 22 & 69 \\
Resilience & $62.48 \pm 18.29$ & 30 & 89 \\
\hline
\end{tabular}

5 S. Jurnal of 
Table 2. Predicting the substance abuse trend of study participants based on resilience and academic procrastination

\begin{tabular}{ccccccccc}
\hline Predictor Variable/ Sources Change & B & SE & $\boldsymbol{\beta}$ & $\mathbf{P}$ & $\mathbf{R}$ & $\mathbf{R 2}$ & Reset R2 & F \\
\hline Substance abuse tendency & & & & & 0.526 & 0.529 & 0.540 & 191.910 \\
Resilience & -0.494 & 0.618 & -0.564 & 0.001 & & & & \\
Academic procrastination & 0.496 & 0.420 & 0.436 & 0.001 & & & & \\
\hline
\end{tabular}

Journal of
Arak University of Medical Sciences

over, resilience $(-0.564)$ and academic procrastination $(-0.436)$ have played significant roles in predicting the investigated students' tendency to abuse drugs (Table2).

\section{Discussion}

The current study aimed to predict the tendency of substance abuse in the second-grade male students based on resilience and academic procrastination. The obtained results indicated a significant and negative relationship between resilience and substance abuse tendency in the investigated students. Besides, resilience predicted the tendency of substance abuse in students.

These data suggest that the higher the students' resilience, the lower their tendency to abuse drugs. These results are consistent with those of Mohammadi, Aghajani et al. [18], and Kennedy, Chen, Fang, and associates [19].

The present study findings also revealed a positive and significant relationship between academic procrastination and substance abuse in male students; academic procrastination predicted the tendency to substance abuse in the sample students. In other words, the higher the procrastination level, the greater the tendency to abuse drugs in the study samples. These data are consistent with those of Tavakoli and Dehkordian [21], and Ferrari and colleagues [22]. Tavakoli and Dehkordian argued that economic status, age, field of study, and academic procrastination explained a total of $13 \%$ of the variance of substance dependence. Moreover, Fugitive and colleagues [22] indicated a relationship between academic procrastination and substance dependence.

\section{Ethical Considerations}

\section{Compliance with ethical guidelines}

This study was approved by the Research Ethics Committee of Kharazmi University (Code: IR.KHU.REC1397.29).

\section{Funding}

According to the authors of the article, there was no financial support for the research.

\section{Authors' contributions}

All authors contributed in preparing this article.

\section{Conflicts of interest}

The authors declared no conflicts of interest.

\section{Acknowledgements}

The authors would like to thank the Education Department of Tehran, District 12, for their cooperation and all those who helped with conducting this study. 


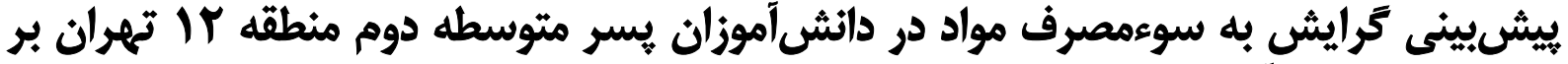

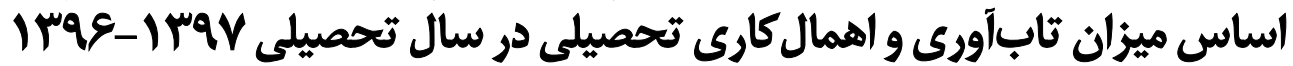

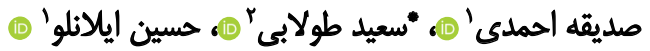 \\ 1. كروه مشاوره و راهنمايى، دانشكده روانشناسى و علوم تربيتى، دانشُكاه خوارزمى، تهران، ايران.

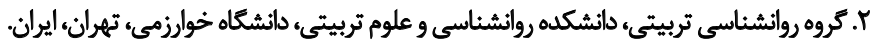

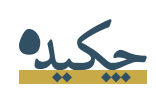

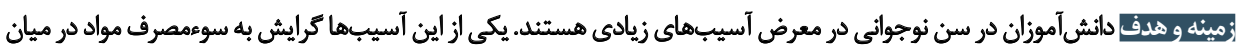

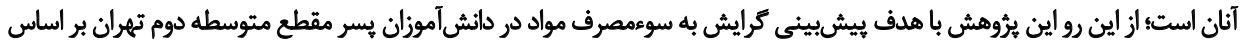

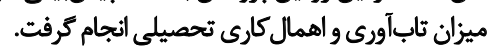

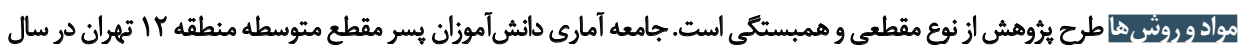

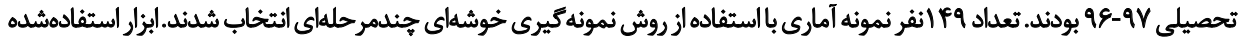

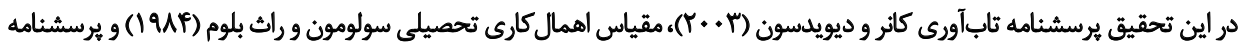

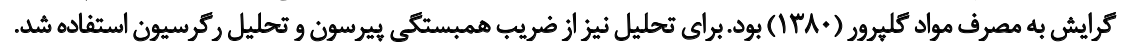

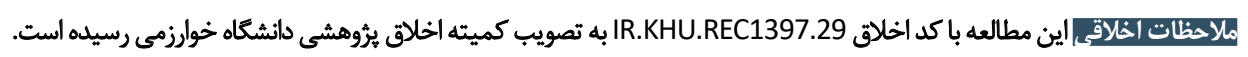

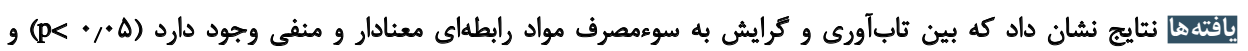

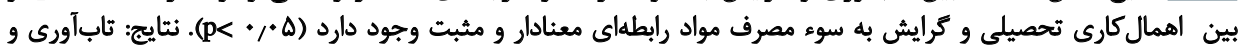

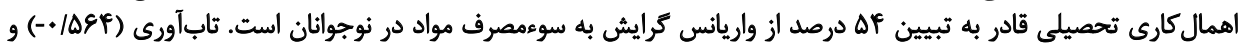

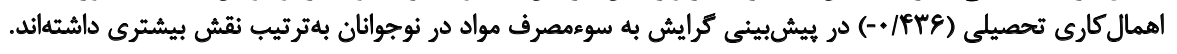

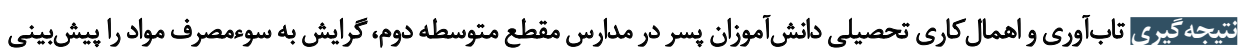

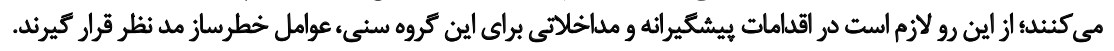

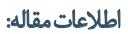

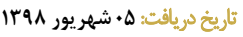

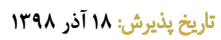
تاريخ اتثشار: تا فروردين

كليدوارهها:

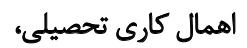

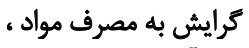
ثاب آورى به مصر

است كه از نظر روانى و جسمى به يك ماده مخدر وابستكى بيدا

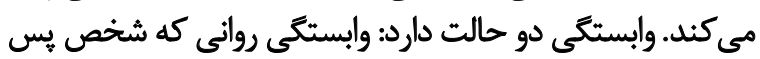

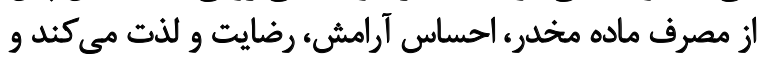

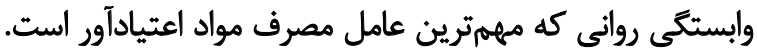

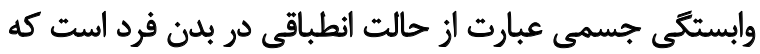

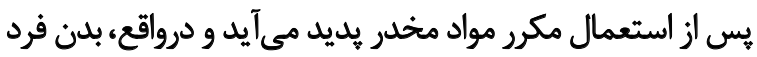

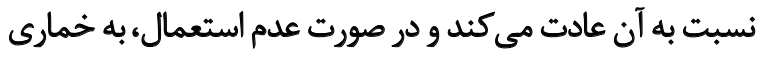

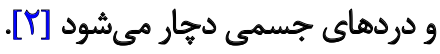

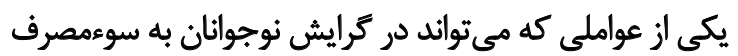

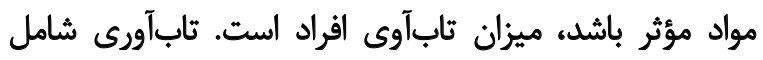

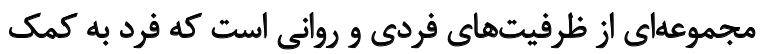

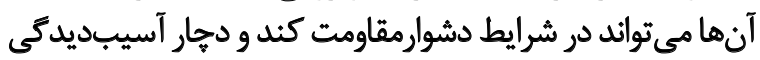

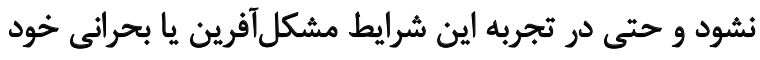

بدون ترديد يكى از مهمترين مسائل بشرى كه ابعاد كسترده

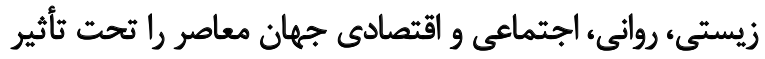

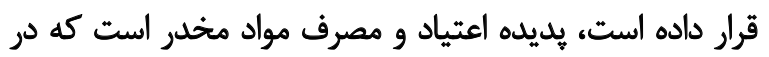

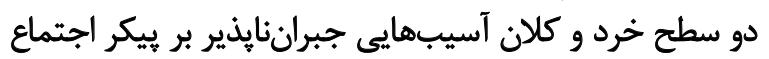

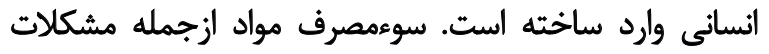

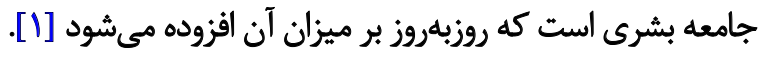

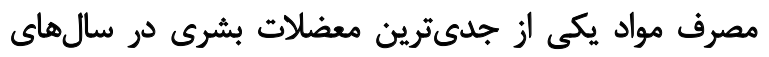

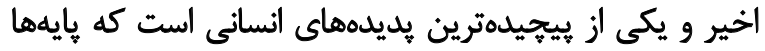

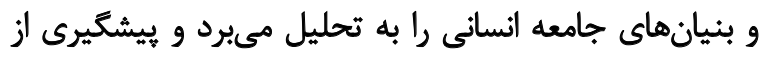

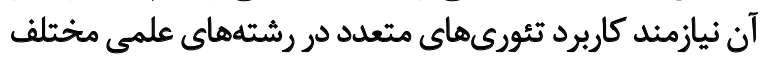

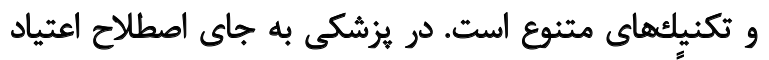

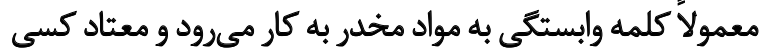




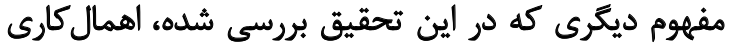

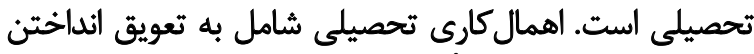

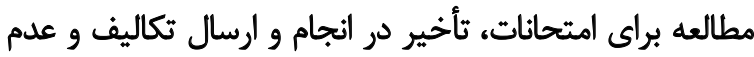

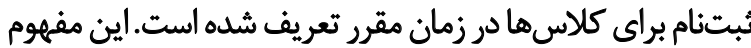

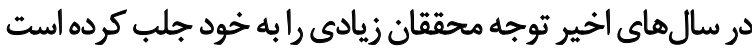

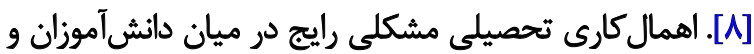

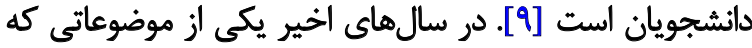

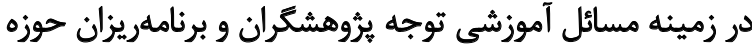

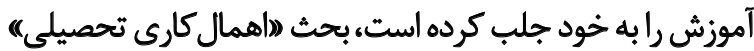

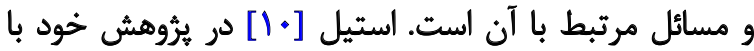

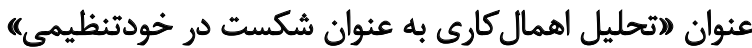

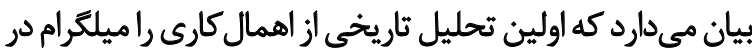

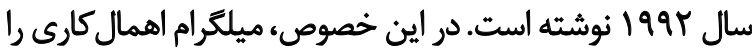

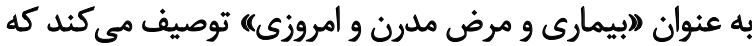

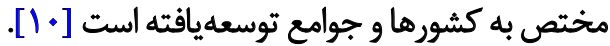

اهمال كارى تحصيلى از مهمترين مسائلى است كه همه ساله به ميزان زيادى امكائات، منابع و استعدادهاى بادي بالقوه انسانى و

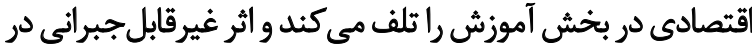

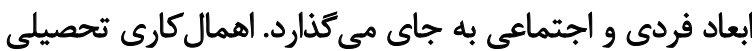

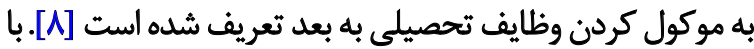

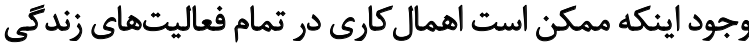

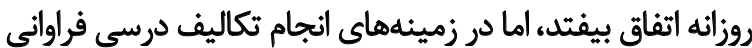

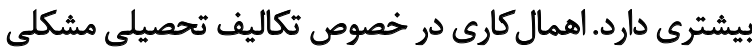

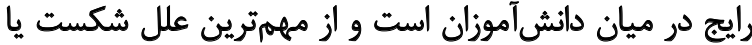

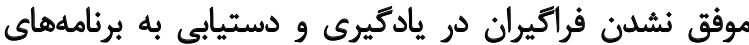
ي يشرفت تحصيلى است.

اهمال كارى تحصيلى با وظايف تحصيلى در ارتباط است و باني

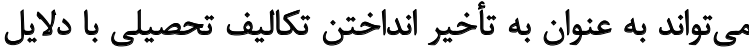

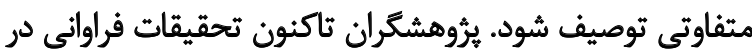

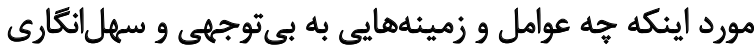

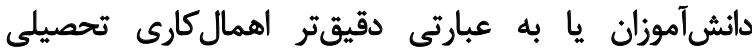

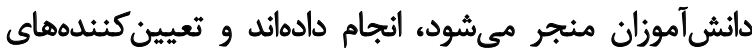

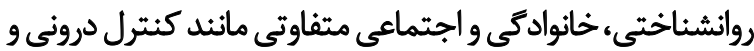
بيرونى، خودكارآمدى عمومى و خودتنظيمي [11] و و سبكهائي

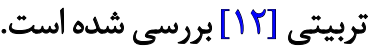

با مطالعه روند اعتياد در كشور مشاهده مى دشود كه ميزان

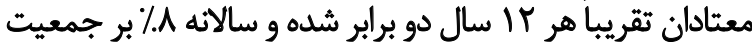

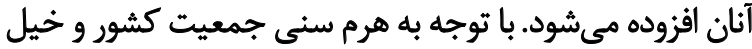

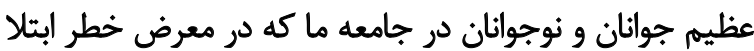

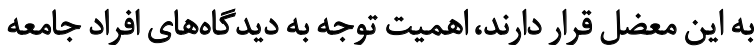

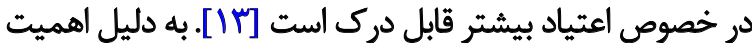

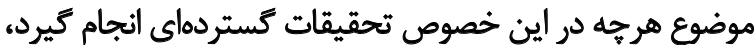

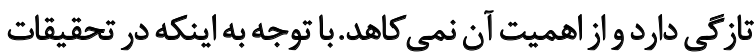

را به لحاظ شخصيتى ارتقاء بخشد. بهعبارتبهتر، تابآورى را

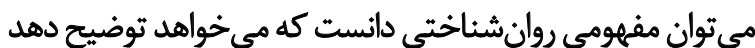

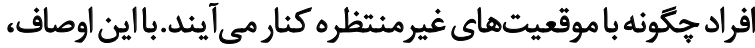

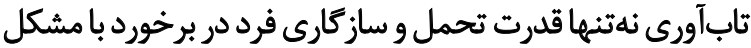

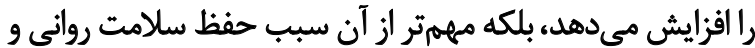

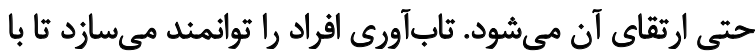

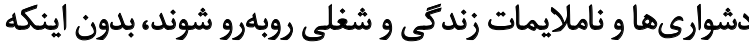

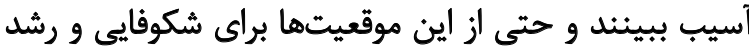

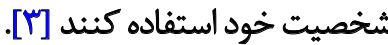

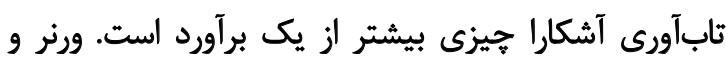

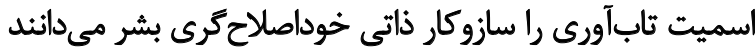

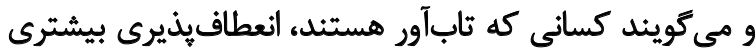

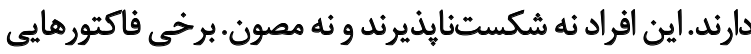

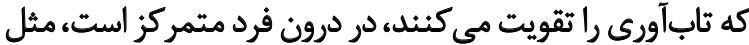

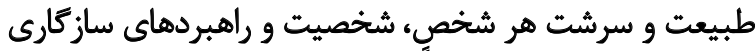

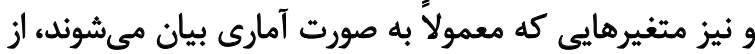

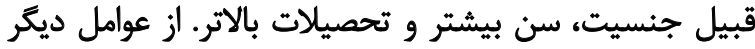

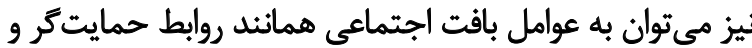
دسترسى به بيوندهاى اجتماعى اشاره كرد [?].

بروكس ثابآورى را ساز كارى مثبت فرد در واكنش به شرايط

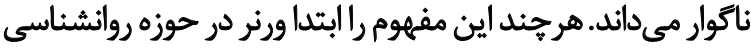

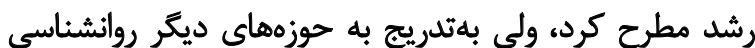

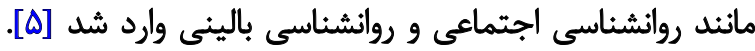

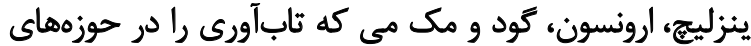

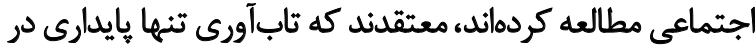

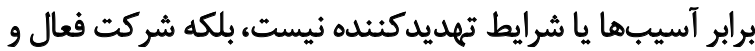

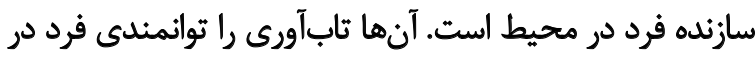

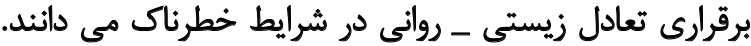

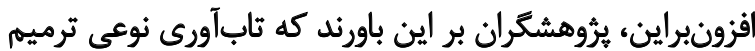

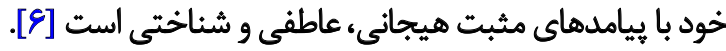
در سالهاى اخير، تابآورى يكى از متغيرهاى نو و مهرم در حوزه

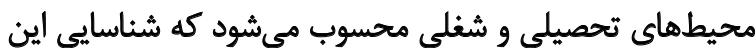

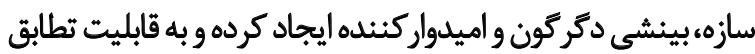

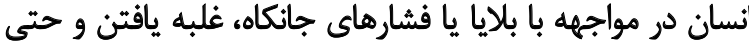

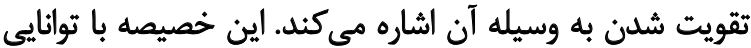

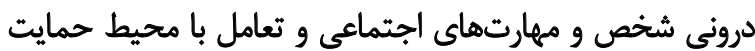

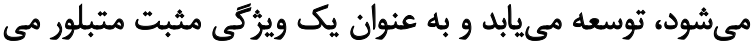

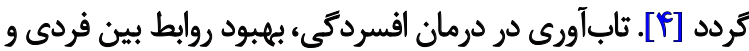
اجتماعي، سلامت روان و تنظيم هيجان مؤثر است و ودر در سال هائي

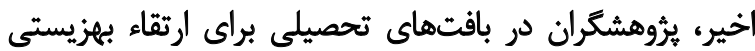

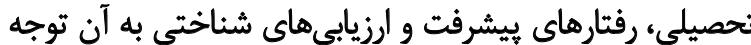

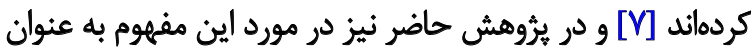

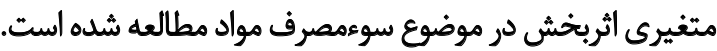




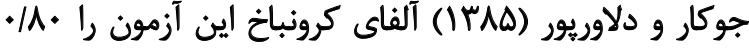

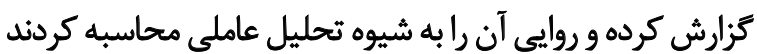

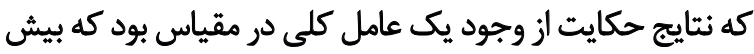

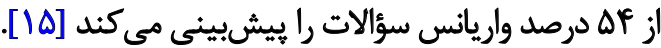

\section{يرسشنامه اهمال كارى تحصيلى سولومون و راث بلوم}

اين مقياس را سولومون و راث بلوم [19]، ساختهاند كه داراى

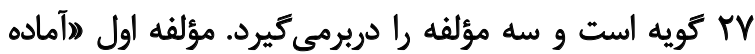

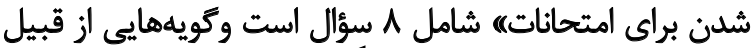

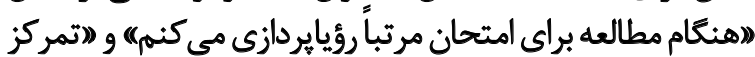

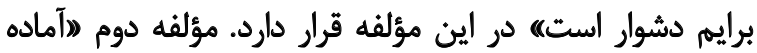

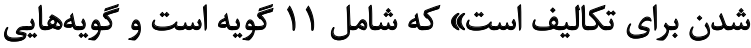

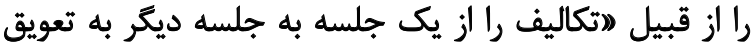

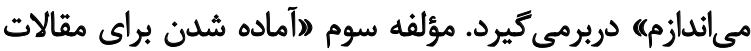

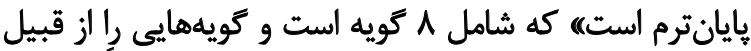
"اهنكامى كه مجبور باشم مقالهاي را آماده كنم، مرتباً آن را با به

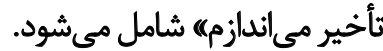

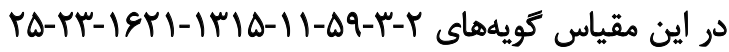

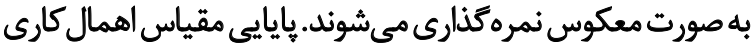
تحصيلى از طريق آلفاى كرونباخ در مطالعهاي كه سولوني الومون انجام

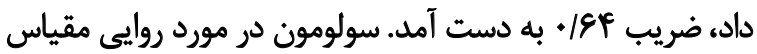

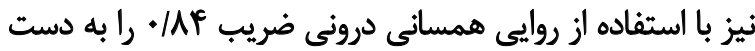

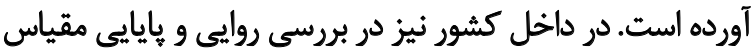

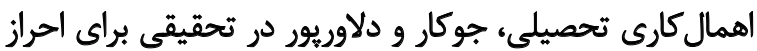

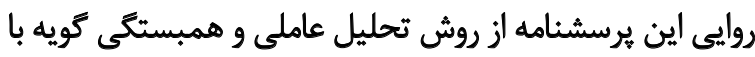

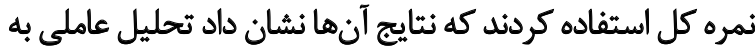

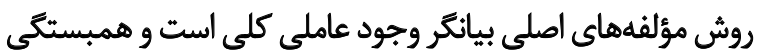

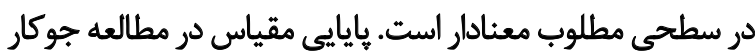

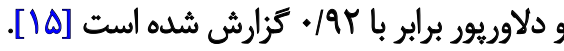

\section{يرسشنامه كرايش به مواد كليرور}

اين يرسشنامه از هf سؤال تشكيل شده است كه تهيه و تنظيم

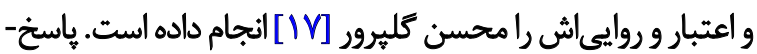

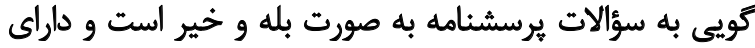

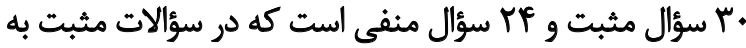

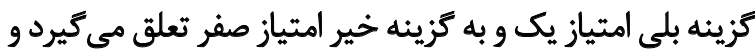

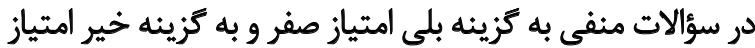

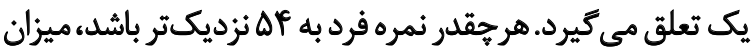

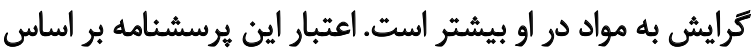

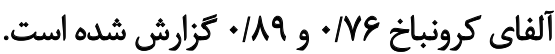

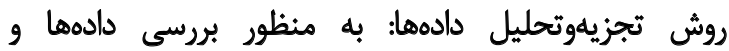

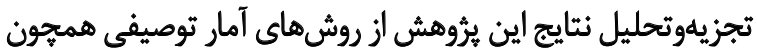

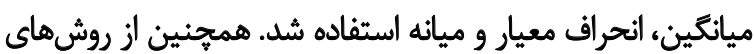

بيشين نقش مؤلفه اهمال كارى تحصيلى و تابآورى در كرايش

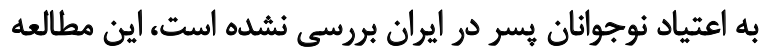

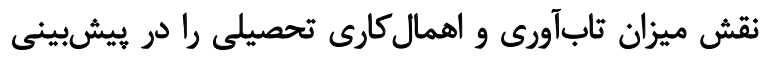

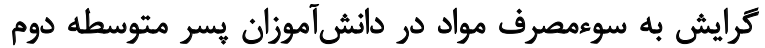
منطقه با تهران بررسى كرده است.

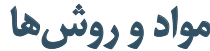

ايزوهش حاضر مطالعهاى مقطعى و تحليلى از نوع همبستى إنى

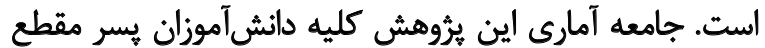

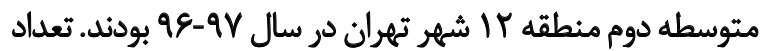

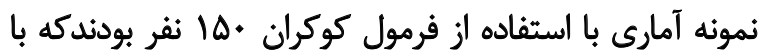

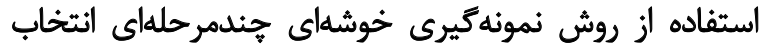

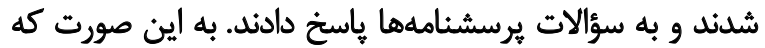

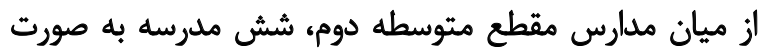

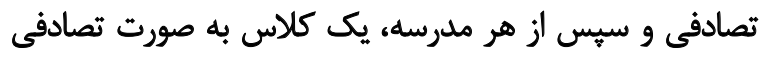

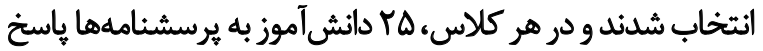

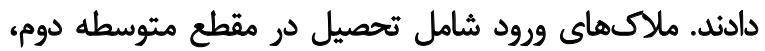

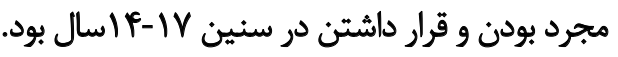

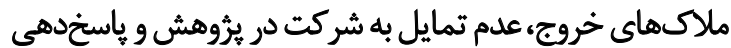

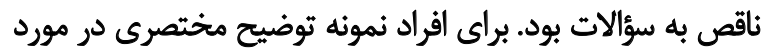

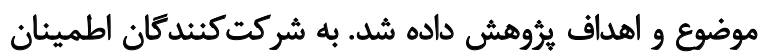

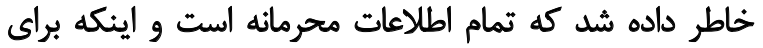

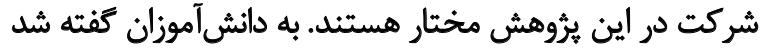

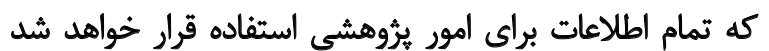

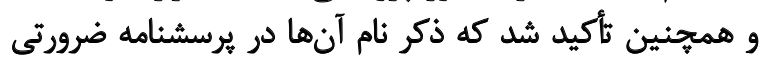

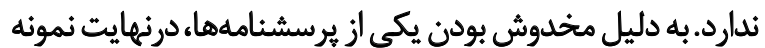

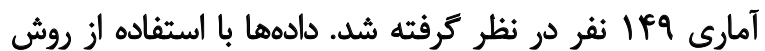

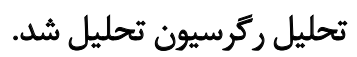

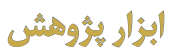

\section{هرسشنامه تاب آورى كانر و ديويدسون}

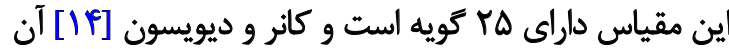

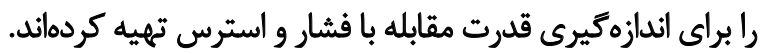

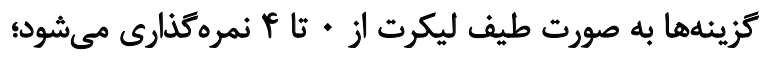

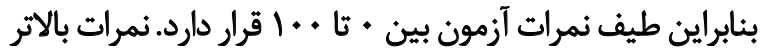

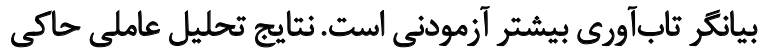

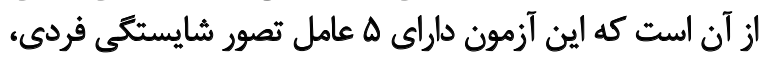

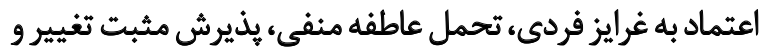
روابط ايمن، كنترل و تأثيرات معنوى استئ.

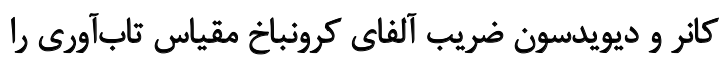

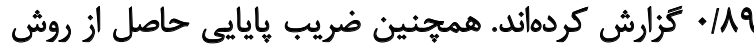

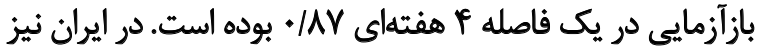


همان كونه كه جدول ضرايب ييشبينى گرايش به سوءمصرف

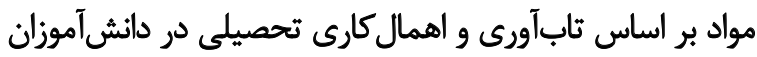

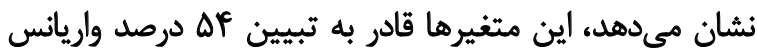

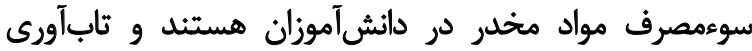
(ئ) كرايش به سوءمصرف مواد در دانشآموزان نقش داشتهاند.

هدف از يُوهش حاضر، يجشبينى گرايش به سوءمصرف مواد

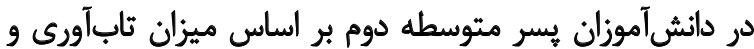

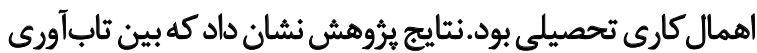

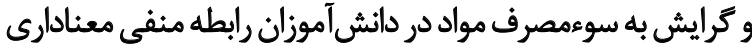

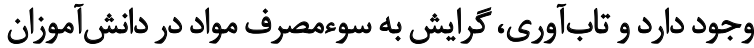

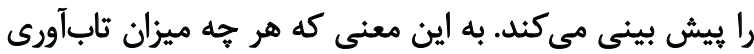

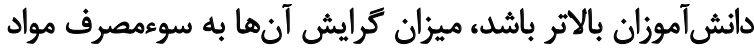

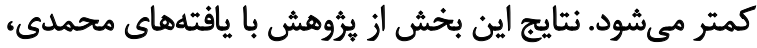

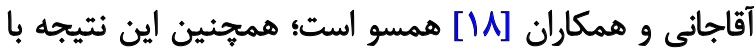

يافتههاى كندى، جن، فنگ و همكاران همسو است [19: در تبيين يافته فوق مىتوان كفت كه افراد تابآور در شرايط

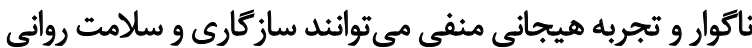

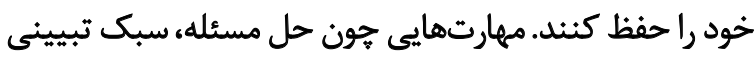

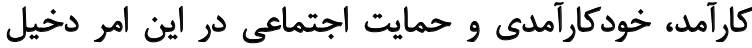

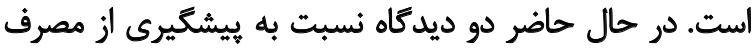

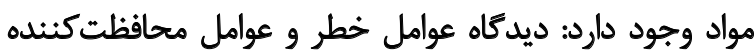

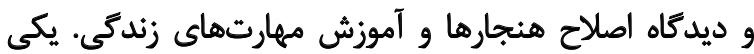

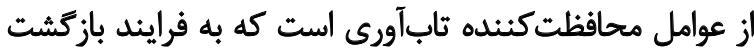

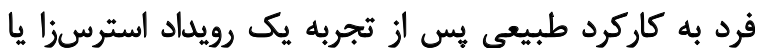

آمار استنباطى مانند آزمون بيرسون و تحليل ركرسيون گام به كام

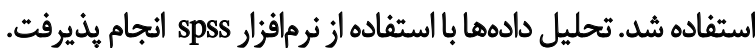

Larịl

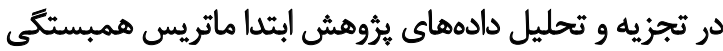

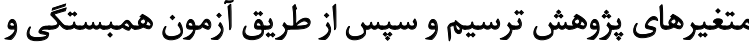

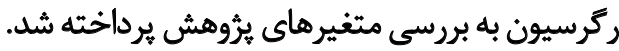

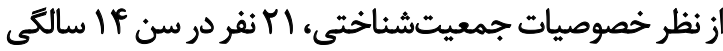

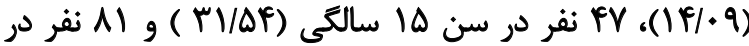

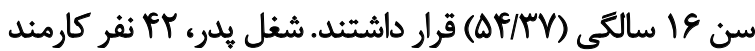
شغاد (TN/A)

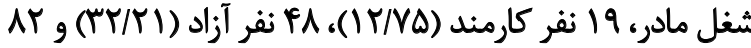

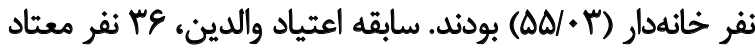

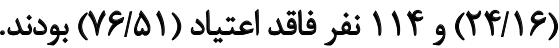

نتايج ضرايب همبستكى متغيرهاى يروهش نشان داد كه ميزان

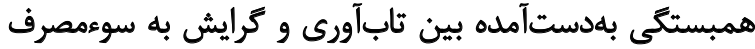

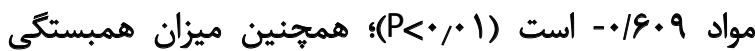

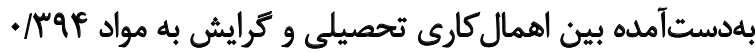

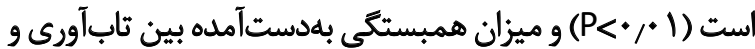

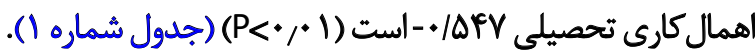

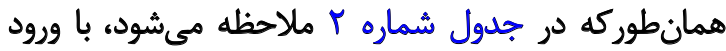

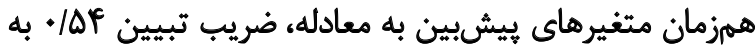

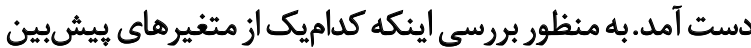

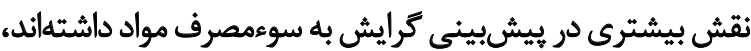

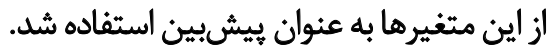

جدول ا. ميانكين و انحراف معيار متغيرهاي كرايش كرايش به سوءمصرف مواد، اهمال كارى تحصيلى و تابآورى

\begin{tabular}{|c|c|c|c|c|}
\hline بيشترين & كمترين & انحراف استاندارد & مياتكين & شاخص متغير \\
\hline rq & r & $N \cdot r$ & $14 / 11$ & كرايش به سوء مصرف مواد \\
\hline Eq & rr & Ir/er & $\Delta r / I F$ & اهمال كارى تحصيلى \\
\hline 19 & r. & Wrq & SYTHA & تاب أورى \\
\hline
\end{tabular}

جدول r. بيش بينى كرايش به سوء مصرف مواد شركت كنيدكان در مطالعه بر اساس ميزان تاب آورى و اهمال كارى تحصيلى

\begin{tabular}{|c|c|c|c|c|c|c|c|c|}
\hline$F$ & RY تنظيم شده RY & RY & $\mathbf{R}$ & $\mathbf{P}$ & $\boldsymbol{\beta}$ & SE & B & متغير ييش يين/ منابع تغيير \\
\hline \multirow[t]{3}{*}{$191 / 91}$. & $. / \Delta)^{e}$ & ./AFq & . larg & & & & & كرايش به سوء مصرف مواد \\
\hline & & & & $\% 1$ &.- IAPF & .1811 & $-* / p q p$ & تاب آورى \\
\hline & & & & .1 .01 & . preg & . Met. &.$/ e q s$ & اهمال كارى تحصيلى \\
\hline
\end{tabular}




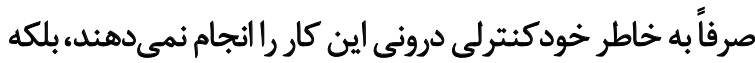

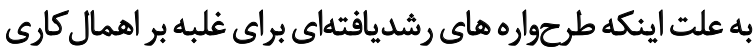
دارند، كمتر اهمال كارند.

دريايان بيشنهاد مىشود راهكارهايي براي ييشكيرى و مقابله با

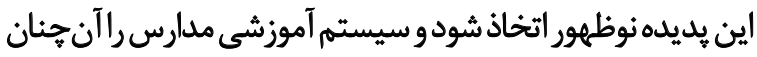

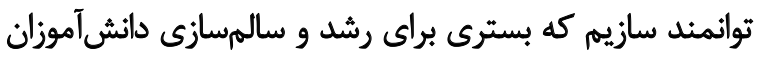

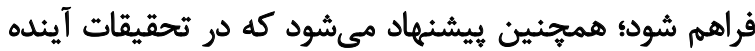

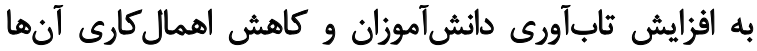

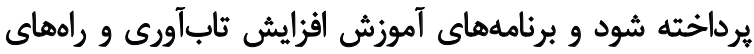

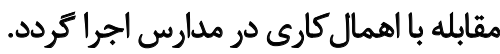

ملاحظات اخلاقى

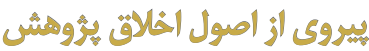

اين مطالعه با كد اخلاق و كميته اخلاق يروهشى دانشكاه خوارزمي رسيده است.

$$
\text { the }
$$

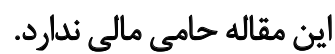

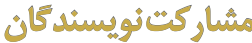

تمامى نويسندكان معيارهاى استاندارد نويسئدىى بر اساس

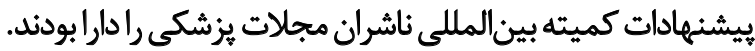

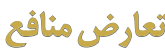

بدينوسيله نويسندكان اعلام ميدارند كه هيجكونه تضاد منافعى در خصوص مطالعه حاضر وجود ندارد.

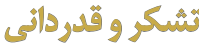

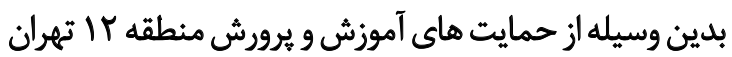

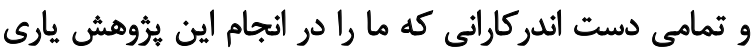
كردند تشكر و قدردانى مي شود.
ناراحت كننده اشاره دارد و ازاينرو افراد تابآور در برابر شرايطى مقاومت

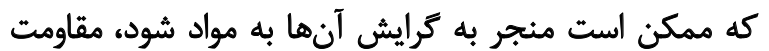

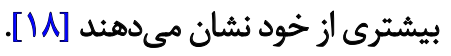

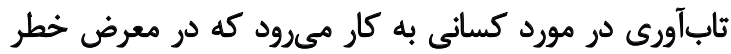

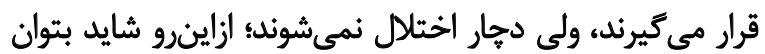

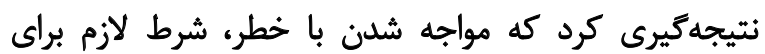

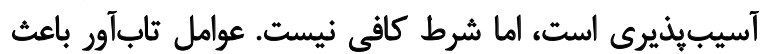

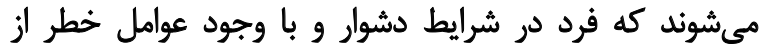

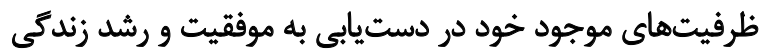

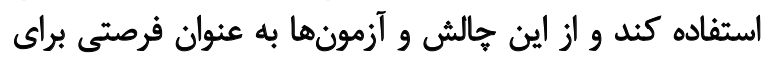

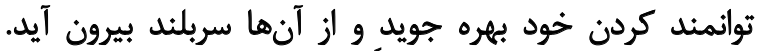

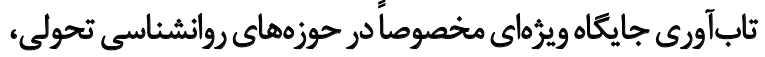

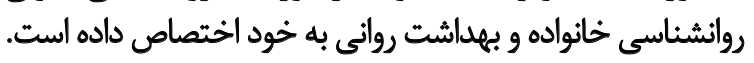

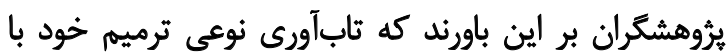

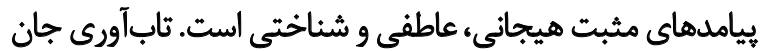

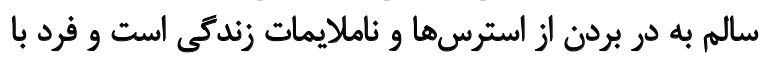

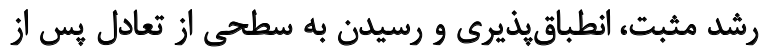

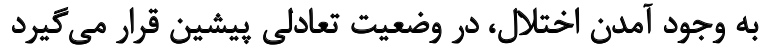

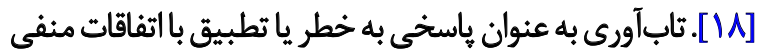

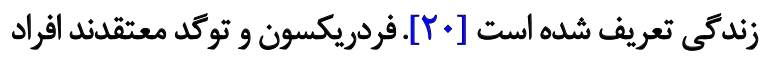

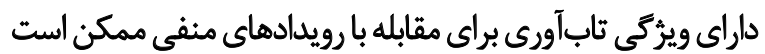

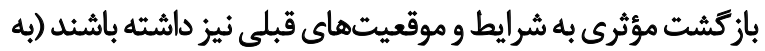

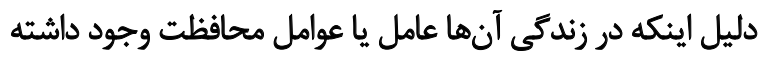

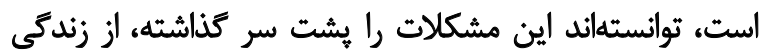

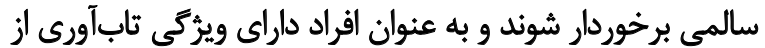

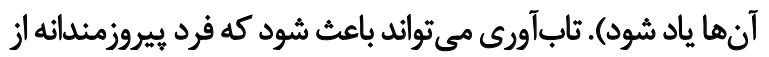

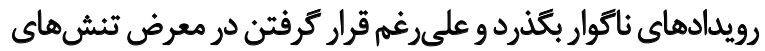
شديد، شايستكى اجتماعى، تحصيلى و شغلى او ارتقاء يابد.

نتيجه ديكر يُروهش اين بود كه بين اهمال كارى تحصيلى و

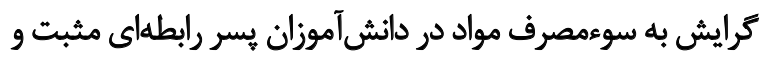

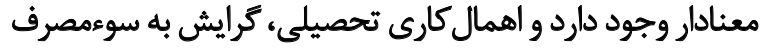

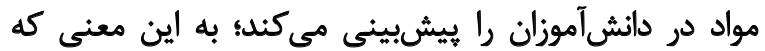

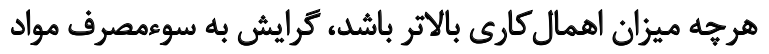

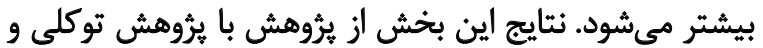

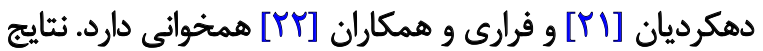

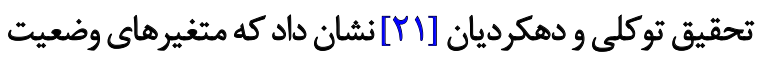

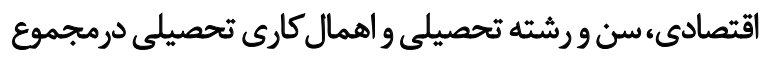

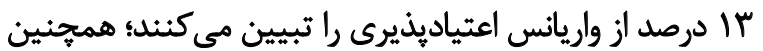

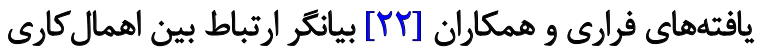

$$
\text { تحصيلى و اعتياديذيرى است. }
$$

نتيجليرى

در تبيين اين يافته مىتوان كفت افرادى كه كمتر اهمال كارند، 


\section{References}

[1] Bahraminezhad A, Beiranvand A, Zare S, Arnavaz M. [The relationship between emotional intelligence and addiction tendency in students of Lorestan University of Medical Sciences (Persian)]. Yafte. 2017; 19(4):113-20. http://eprints.lums.ac.ir/1074/

[2] Siam Sh. Drug abuse prevalence between male students of different universities in rasht in 2005. Zahedan J Res Med Sci. 2007; 8(4):279-85.

[3] Pour Sardar F, Abbaspour D. The effect of resilience on mental health and life satisfaction, a psychological pattern of well-being. Quarterly Journal of New Thoughts in Educational Sciences 2012; 14(1):81-9.

[4] Haghghizadeh M. [The relationship between resilience and spiritual intelligence with mental health of students of Islamic Azad University of Khorramabad (Persian)] [MA. thesis]. Garmsar: Islamic Azad University, Garmsar Branch; 2015. http://opac.nlai.ir/opac-prod/bibliographic/3960297

[5] Brooks BA, Anderson MA. Defining quality of nursing work life. Nurs Econ. 2005; 23(6):319-26. [PMID]

[6] Inzlicht M, Aronson J, Good C, McKay L. A particular resiliency to threatening environments. J Exp Soc Psychol. 2006; 42(3):323-36. [DOI:10.1016/j.jesp.2005.05.005]

[7] Toulabi S, Hasanabadi HR, Izanloo B, Rahiminejad SP. [The effect of resilience program training on cognitive appraisal and achievement behaviors among university students (Persian)]. J Cogn Psychol. 2017; 5(3):5769. http://jcp.khu.ac.ir/article-1-2699-en.html

[8] Toker B, Avci R. Effect of cognitive-behavioral-theory-based skill training on academic procrastination behaviors of university students. Educ Sci Theory Pract. 2015; 15(5):1157-68. https://www.researchgate.net/ publication/288516693

[9] XuZ.Just doit! Reducing academic procrastination of secondarystudents. Interv Sch Clin. 2016; 51(4):212-9. [DOI:10.1177/1053451215589178]

[10] Steel P. The nature of procrastination: A meta-analytic and theoretical review of quintessential self-regulatory failure. Psychol Bull. 2007; 133(1):65-94. [DOI:10.1037/0033-2909.133.1.65] [PMID]

[11] Senécal C, Koestner R, Vallerand RJ. Self-regulation and academic procrastination. J Soc Psychol. 1995; 135(5):607-19. [DOI:10.1080/002245 45.1995.9712234

[12] Jaradat AM. Test anxiety in Jordanian students: Measurement, correlates and treatment [PhD. dissertation]. Marburg/Lahn: Philipps-University Marburg; 2004. https://pdfs.semanticscholar.org/9133/fffaf773bd681d8be09eeec06bba66ba6fd0.pdf

[13] Meysamie AP, Faramarzi B, Holakouie Naieni K. [How addicts think about addiction and community problems? (Persian)]. Tehran Univ Med J. 2006; 64(5):34-43. https://www.sid.ir/fa/journal/ViewPaper. aspx?ID=51100

[14] Connor KM, Davidson JR. Development of a new resilience scale: The Connor-Davidson Resilience Scale (CD-RISC). Depress Anxiety. 2003; 18(2):76-82. [DOI:10.1002/da.10113] [PMID]

[15] Jokar B, Delavarpour M. [The relationship between Academic procrastination and goal achievement (Persian). Journal of New thoughts on Education. 2007; 3(3):61-80. [DOI:10.22051/JONTOE.2007.312]

[16] Solomon L, Rothblum ED. Academic procrastination: Frequency and cognitive-behavioral correlates. J Couns Psychol. 1984; 31(4):503-9. [DOI:10.1037/0022-0167.31.4.503]
[17] Golparvar M, Molavi H. [Comparison of psychological and marital relationships between addicts and non-addicts with their husbands (Persian)]. Knowl Res Appl Psychol. 2001; (7):1-20. http://ensani.ir/fa/ article/17886/

[18] Mohammadi A, Aghajani M, Zahtabvar GH. [Addiction and its relation with resilience and emotional components (Persian)]. Iran J Psychiatr Clin Psychol. 2011; 17(2):136-42. http://ijpcp.iums.ac.ir/article1-1347-en.html

[19] Kennedy B, Chen R, Fang F, Valdimarsdottir U, Montgomery S, Larsson $\mathrm{H}$, et al. Low stress resilience in late adolescence and risk of smoking high alcohol consumption and drug use later in life. J Epidemiol Community Health. 2019; 73(6):496-501. [DOI:10.1136/jech-2018-211815] [PMID]

[20] Newman JL, Dantzler J. Fostering individual and school resilience: When students at risk move from receivers to givers. J Community Engagem Scholarsh. 2019; 8(1):9. https://digitalcommons.northgeorgia. edu/cgi/viewcontent.cgi?article=1266\&context=jces

[21] Tavakoli MA, Dehkordian P. [Prediction of university student's addictability based on some demographic variables, academic procrastination, and interpersonal variables (Persian)]. Res Addict. 2014; 7(28):23-41. http://etiadpajohi.ir/article-1-277-en.html

[22] Ferrari JR, Johnson JL, McCown WG. Procrastination and task avoidance: Theory, research, and treatment. Boston, MA: Springer-Verlag; 1995. [DOI:10.1007/978-1-4899-0227-6] 
This Page Intentionally Left Blank 